\title{
Deletion of Exon 1 in AMER1 in Osteopathia Striata with Cranial Sclerosis
}

\author{
Jingyi Mi ${ }^{1}$, Padmini Parthasarathy ${ }^{1}$, Benjamin J. Halliday $\left.{ }^{1}{ }^{(}\right)$, Tim Morgan ${ }^{1}$, John Dean ${ }^{2}{ }^{(}$, \\ Malgorzata J. M. Nowaczyk ${ }^{3}$, David Markie ${ }^{4}$, Stephen P. Robertson ${ }^{1, *}$ and Emma M. Wade ${ }^{1(D)}$ \\ 1 Department of Women's and Children's Health, Dunedin School of Medicine, University of Otago, \\ Dunedin 9016, New Zealand; mije6460@student.otago.ac.nz (J.M.); mini.parthasarathy@otago.ac.nz (P.P.); \\ halbe104@student.otago.ac.nz (B.J.H.); tim.morgan@otago.ac.nz (T.M.); emma.wade@otago.ac.nz (E.M.W.) \\ 2 North of Scotland Regional Genetics Service, NHS Grampian, Ashgrove House, Foresterhill, \\ Aberdeen AB25 2ZA, UK; j.dean@abdn.ac.uk \\ 3 Department of Pathology and Molecular Medicine, Health Sciences Centre, McMaster University, \\ Hamilton, ON L8S 4K1, Canada; nowaczyk@hhsc.ca \\ 4 Department of Pathology, Dunedin School of Medicine, University of Otago, Dunedin 9016, New Zealand; \\ david.markie@otago.ac.nz \\ * Correspondence: stephen.robertson@otago.ac.nz; Tel.: +64-(0)3-479-7469
}

Received: 3 November 2020; Accepted: 26 November 2020; Published: 30 November 2020

\begin{abstract}
Osteopathia striata with cranial sclerosis (OSCS) is an X-linked dominant condition characterised by metaphyseal striations, macrocephaly, cleft palate, and developmental delay in affected females. Males have a more severe phenotype with multi-organ malformations, and rarely survive. To date, only frameshift and nonsense variants in exon 2, the single coding exon of AMER1, or whole gene deletions have been reported to cause OSCS. In this study, we describe two families with phenotypic features typical of OSCS. Exome sequencing and multiplex ligation-dependent probe amplification (MLPA) did not identify pathogenic variants in AMER1. Therefore, genome sequencing was employed which identified two deletions containing the non-coding exon 1 of AMER1 in the families. These families highlight the importance of considering variants or deletions of upstream non-coding exons in conditions such as OSCS, noting that often such exons are not captured on probe or enrichment-based platforms because of their high $\mathrm{G} / \mathrm{C}$ content.
\end{abstract}

Keywords: AMER1; WTX; osteopathia striata with cranial sclerosis

\section{Introduction}

Osteopathia striata with cranial sclerosis (OSCS: MIM\# 300373) is a rare skeletal dysplasia caused by loss-of-function or truncating variants in, or deletion of, AMER1 (MIM\# 300647; also known as WTX/FAM123B) [1-3]. OCSC has an X-linked, dominant mode of inheritance and, in females, is primarily characterised by metaphyseal striations, osteosclerosis, macrocephaly, hypertelorism and occasionally, developmental delay. Affected males rarely survive the perinatal period; the occasional survivor exhibits osteosclerosis without metaphyseal striations, as well as cardiovascular, gastrointestinal, and genitourinary malformations, alongside features present in typically affected females. Males with mosaicism for loss of function AMER1 variants, or those with Klinefelter syndrome (XXY), manifest a phenotype similar to female heterozygotes [4].

AMER1 encodes adenomatous polyposis coli (APC) membrane recruitment protein 1, which regulates the WNT1 signalling pathway by stimulating $\beta$-catenin degradation. Loss-of-function variants resulting in reduced expression of AMER1 cause enhanced WNT signalling through increased nuclear accumulation of $\beta$-catenin [5]. WNT signalling is involved in cellular differentiation and proliferation during embryogenesis 
and tumorigenesis, hence its loss results in a pleiotropic clinical phenotype. Increased WNT-signalling causes enhancement of osteoblast maturation and function [6,7], leading to increased bone density [8].

In this report, we describe two families with OSCS phenotypes who were found to have novel pathogenic variants in AMER1, (NM_152424.4:c.-417_-99+1606del) and (NM_152424.4:c.-1290_-99+380del), resulting in isolated deletion of the $5^{\prime}$ non-coding exon 1 . Previous attempts at a diagnosis using exome sequencing and multiplex ligation-dependent probe amplification (MLPA) did not detect the deletions because these platforms did not target this non-coding exon for sequencing and dosage analysis respectively. The deletions were subsequently discovered using genome sequencing (GS). These cases highlight the importance of screening $5^{\prime}$ untranslated exons in genes that produce phenotypes due to a loss-of-function mechanism.

\section{Materials and Methods}

\subsection{Subjects and Ethical Consent}

Two unrelated individuals with OSCS were ascertained by physician-initiated referral. The study was conducted in accordance with the Declaration of Helsinki and families were consented to participate in a research study under approved protocols MEC/08/08/094 and 13/STH/56 (Health and Disability Ethics Committee, Dunedin, New Zealand). Clinical images are reproduced with consent from the families.

\subsection{Sequence Analysis}

Genomic DNA was extracted from blood from the affected individuals and their family members. $\mathrm{X}$-inactivation analysis, and multiplex ligation-dependent probe (MLPA) analysis and targeted Sanger sequencing of the AMER1 coding exon were performed as described previously [9].

Exome sequencing was performed by Otogenetics (Atlanta, GA, USA), and genome sequencing was performed by the Kinghorn Centre for Clinical Genomics (Sydney, Australia) using a HiSeq2500 (Illumina, San Diego, CA, USA). Exome enrichment was performed with the Agilent SureSelect Human All Exon V4+UTRs (untranslated regions) or V5+UTRs capture kits (Agilent Technologies, Santa Clara, CA, USA). Preparation for genome sequencing was performed with the Illumina 30x GS (TruSeq Nano (Illumina)) v2.5 kit. Reads were paired, with a read length of 150 base pairs. Alignment of reads and variant calling was done according to the Genome Analysis Tool Kit (GATK) best practice guidelines [10,11]. Alignment to the reference sequence (GRCh37 assembly) was performed with the Burrows-Wheeler Aligner (v0.7.17) with the MEM algorithm [12]. Picard MarkDuplicates (v2.18.11) was used to identify duplicate reads [13] and aligned files in bam format were produced using GATK (v3.8) BaseRecalibrator. GATK HaplotypeCaller was used to generate GVCFs, containing single nucleotide variants (SNVs) and short insertions/deletions (indels). Multi-sample VCFs were produced using GATK GenotypeGVCFs, which were annotated with gene context information using SnpEff (v4.3S) [14]. Population allele frequencies from the gnomAD project (v2.1.1) [15] were added using BCFtools annotate (v1.9).

Manta (v1.6.0) [16] was used to call large deletions and duplications using the genome sequences, as well as inversion and insertion events and SnpEff was used to annotate the structural events for genic impact, in VCF format. Data from the Database of Genomic Variants (DGV, release date 15 May 2016) was used to identify novel structural events.

Allele specific PCR using genomic DNA extracted from dermal fibroblasts confirmed segregation of the variant within the families. PCR was performed with Q5 HotStart High Fidelity DNA polymerase (NEB, Ipswich, MA, USA). Individual PCR primers were designed for the two families, but the experimental design was the same. Primer set 1 was designed to flank the deletions, producing products too large for amplification under the chosen conditions from the WT allele, therefore amplifying only the deletion-containing allele, producing products of $1221 \mathrm{bp}$ (Family A) or $546 \mathrm{bp}$ (Family B). Primer set 2 consists of one primer embedded within the deletion, amplifying only the 
wild-type allele, producing products of 662 bp (Family A) or 669 bp (Family B). PCR conditions were appropriate for amplification of 100-1200 bp products. Primer sequences are available upon request.

\section{Results}

\subsection{Clinical Descriptions}

\subsubsection{Proband A}

Proband A was diagnosed with bilateral cleft lip and palate, and bilateral absence of fibulae on prenatal ultrasound at 26th week gestation. She was born at 30 weeks and 4 days. She had an abnormal head shape, hypertelorism, bilateral cleft lip and palate, and bilateral choanal atresia. She also manifested severe features more commonly seen in the male OCSC phenotype including ventricular septal defect, a secundum atrial septal defect, patent ductus arteriosus, hydronephrosis, intestinal malrotation, and anal stenosis. She required a tracheostomy for laryngotracheomalacia. Magnetic Resonance Imaging (MRI) of the head showed hypoplasia of the splenium of the corpus callosum. Radiographs showed generalized increased bone density, absent fibulae bilaterally, and shortening and undermodelling of the proximal phalanges (Figure 1A-C). Chromosomal microarray demonstrated a normal female chromosomal complement. A clinical diagnosis of OSCS was made in the proband. Her father had a phenotype that included metaphyseal striations, generalised skull osteosclerosis, macrocephaly, coarse facial features, and thickening of the nasal bridge.

\subsubsection{Proband B}

Proband B was born at 35 weeks gestation by caesarean section because of polyhydramnios. Bilateral cleft lip and palate, macrocephaly, hypertelorism, and lymphoedema were noted at birth. She had a nasopharyngeal airway inserted because of upper airway obstruction. She had a bilateral cleft lip repair at 1 year 11 months, and cleft palate repair at 2 years 6 months. A skeletal survey demonstrated metaphyseal striations (Figure 1D) with calvarial thickening. There was no family history. A clinical diagnosis of OSCS was made. Although she had delayed speech initially, her neurodevelopment was subsequently within normal limits, and at age 7 years, she is making normal progress in mainstream education, but continues to need nocturnal continuous positive airway pressure (CPAP).

\subsection{Non-Coding Deletions in AMER1 Cause OSCS}

AMER1 (Xq11.2) contains 2 exons. Exon 1 is non-coding and exon 2 contains the translation initiation site for the coding region. In proband A, Sanger sequencing and MLPA analysis of the single coding exon of $A M E R 1$ was performed, and no pathogenic variants were found. There was no skewing of the ratio of X-chromosome inactivation (54:46). The same sequence and MLPA analysis of AMER1 was performed in her father and demonstrated no anomalies. Similarly, in proband B, no pathogenic variants were found by Sanger sequencing or MLPA analysis. The absence of discoverable variants in $A M E R 1$ led us to seek pathogenic variants at another locus and whole exome sequencing was carried out. This approach failed to identify plausible candidate variants. Since the exon capture platforms used here did not include the first non-coding exon in AMER1, and due to the potential for locus heterogeneity underlying OSCS, whole genome sequencing was utilised. In both probands, a deletion of the $5^{\prime}$ non-coding exon which encodes most of the $5^{\prime}$ untranslated region of AMER1 was identified. Proband A was heterozygous for a 1924 bp deletion, (NM_152424.4:c.-417_-99+1606del, NC_000023.10:g.63423844_63425768del), encompassing the entirety of exon 1 (Figure 1B). PCR analysis (Figure 1C,D) showed that the paternal grandmother, paternal aunt and mother amplified the reference allele. The proband and her father produced both a product amplified from the reference allele and a product amplified by the internal primers (deletion allele). This indicates that the proband has one deletion-containing and one reference allele present. Given that both PCR reactions produced products 
in the father (A-II-2), it is concluded that the deletion arose early in his development rendering him mosaic for the variant which he then transmitted to the proband.
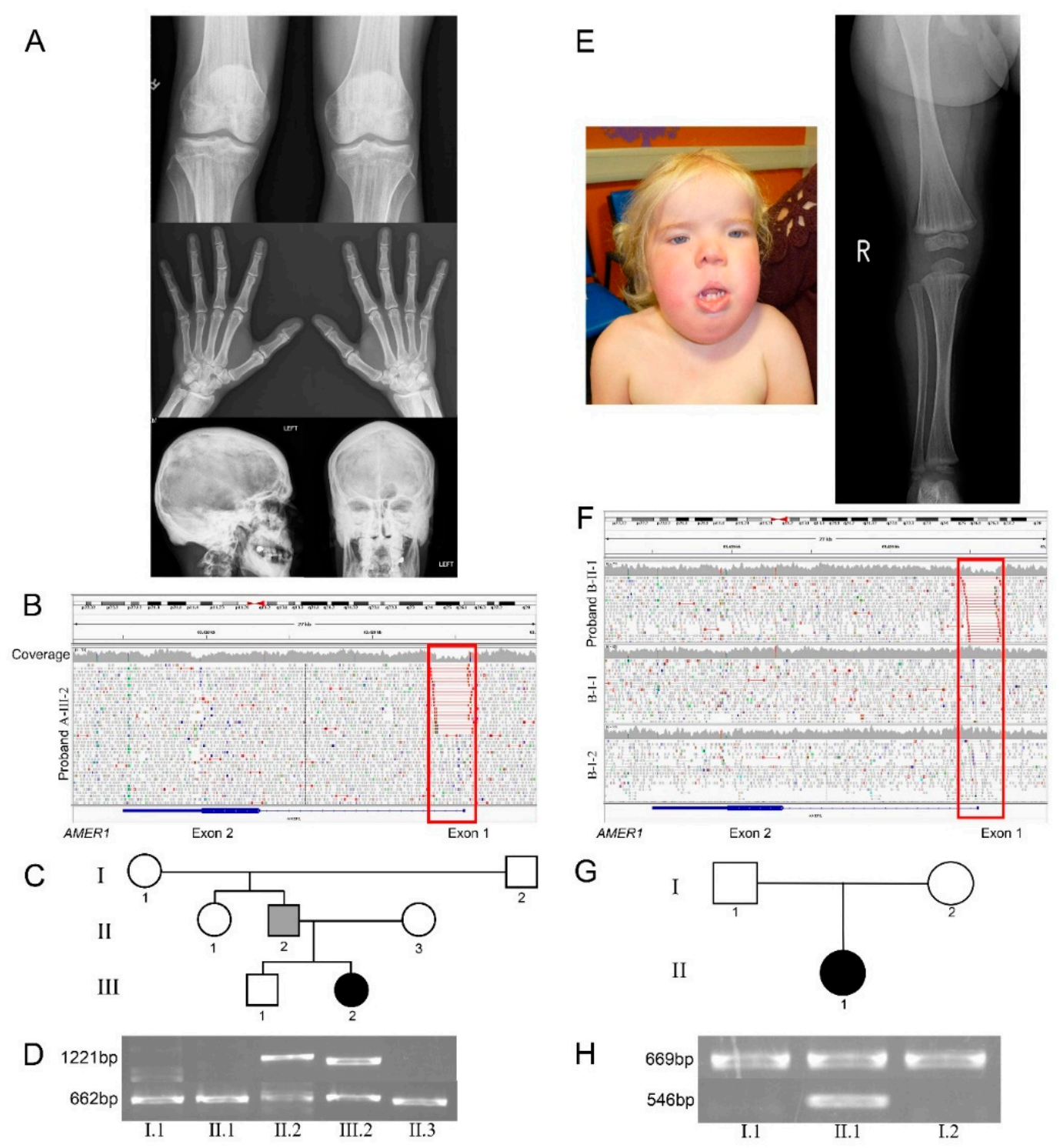

Figure 1. Clinical and sequence data from families A and B. (A) Radiographs from the proband of family A showing metaphyseal striations, cranial sclerosis, and osteosclerosis in the proband; (B) Integrated genome viewer (IGV) screen-shot illustrating the deletion NC_000023.10:63423844_63425768, encompassing exon 1, in the proband (red box). (C) Pedigree and (D) PCR results from family A showing individuals I-1, II-2 and II-3 have a band of 662 bp corresponding to DNA amplified by primer pair 2, the proband III-2 and her father II.2 have bands of both $662 \mathrm{bp}$ and $1221 \mathrm{bp}$ corresponding to DNA amplified by both sets of primers. (E) Clinical image and radiographs from the proband of family B showing macrocephaly, hypertelorism and metaphyseal striations. (F) An IGV screen-shot showing a de novo deletion NC_000023.10:63425070_63426641 in the proband (red box). (G) Pedigree and $(\mathbf{H})$ PCR results are consistent with both parents being unaffected, each carrying only the wild type allele and producing only the primer set 2 product ( $669 \mathrm{bp}$ ), II-1 is heterozygous for a de novo deletion with 2 bands indicating amplification by both primer sets (primer set 1: 546 bp, primer set 2: $669 \mathrm{bp}$ ).

Proband B was shown to be heterozygous for a de novo 1571 bp deletion in AMER1, (NM_152424.4:c.-1290_-99+380del, NC_000023.10:g.63425070_63426641del), encompassing the entirety of exon 1 that encodes most of the $5^{\prime}$ untranslated region of $A M E R 1$ (Figure 1F). PCR results (Figure 1G,H) 
confirmed that both parents only produced the reference product, indicating that they are hemizygous and homozygous, respectively, for the reference allele. The proband (B-II-1) amplified products for both pair of primers, indicating that she carries both a reference and the deletion-containing allele. The pathogenic variant therefore arose de novo in the proband. These variants were not detected using MLPA because a probe pair was not designed for this region of the gene.

Both deletions encompass the entirety of exon 1 (chrX:63,425,624_63,425,450, GRCh37.p13), delete the sole transcription start site for the gene, and predict a loss of transcript produced from this allele. Both have been assigned 'Pathogenic' as per American College of Medical Genetics guidelines (PVS1, PM2, PM4, PP1, PP3 and PP4) [17] and have been submitted to ClinVar (accessions SCV001426685 and SCV001426686, respectively).

\section{Discussion}

Germline truncating variants in AMER1, as well as whole gene deletions, cause OSCS [3,9]. In this study, two different deletions of the $5^{\prime}$ non-coding exon were found in two families. In family A, the deletion segregated with the phenotype; in family B, the deletion arose de novo in the proband. Possible mechanisms of pathogenicity could include: deletion of the transcription start site such that RNA polymerase cannot bind and initiate transcription, or deletion of the splice sites flanking exon 1 resulting in abnormal transcript maturation. In both instances, functional protein will not be produced and both alleles can be considered to be null.

Despite being non-coding, loss of $A M E R 1$ exon 1 results in the same phenotype as loss-of-function variants in the coding region of $A M E R 1$, or whole-gene deletions of the locus [9]. The pathogenic deletions were not discovered by conventional diagnostic methods. Initial Sanger sequencing of AMER1 did not target exon 1 as variants in this region had not been previously implicated in OSCS. MLPA did not detect the deletions, because of an inability to locate a probe pair in such as small sized exon due to its sequence composition. In whole exome sequencing, the $5^{\prime} A M E R 1$ non-coding exon was not represented on the capture platforms used in this study and therefore not sequenced. However, it should be noted that more recent capture kits that capture UTRs do target AMER1 exon 1, for example Agilent SureSelect Human All Exon V6/V7+UTRs, however the high G/C content may still prevent satisfactory sequencing of the region.

These two cases demonstrate the need to conduct similar, focused searches in other genes associated with loss-of-function phenotypes that contain upstream non-coding exons. Deletions that encompass $5^{\prime}$ non-coding exons may be frequently overlooked in conventional diagnostic methods for a number of reasons. Upstream, non-coding exons are often small, therefore deletions that remove them can also be small and likely to be missed by large-scale genomic methods to detect copy number variants. They are also commonly separated from the first coding exon by large introns, therefore targeted sequencing of the whole gene may not extend to these regions, or they will not be captured in some exome sequencing protocols. Furthermore, the frequently high G/C content of these regions complicates the design of efficient capture probes for exome or MLPA type-analyses. Consequently, although focused sequencing may detect single nucleotide variants in $5^{\prime}$ UTRs, a targeted approach to identify pathogenic deletions outside of the coding region should also be considered.

\section{Conclusions}

In conclusion, we identified two novel variants in $A M E R 1$, leading to deletion of the $5^{\prime}$ non-coding exon, in two families with OSCS. This demonstrates that the whole gene should be considered when screening for OSCS, not merely the coding region of exon 2. Inclusion of methodologies such as whole genome sequencing and PCR can increase diagnostic sensitivity in conditions with similar circumstances to what we describe here for OSCS.

Author Contributions: J.M. designed and performed the molecular tests and wrote the manuscript; P.P., B.J.H., and D.M. designed and performed the exome and genome sequencing analysis; T.M. carried out the MLPA and some PCR assays; J.D. supplied clinical information on Proband B; M.J.M.N. supplied clinical information on 
Family A; S.P.R. and E.M.W. supervised the above, prepared the manuscript, and supplied funding. All authors have read and agreed to the published version of the manuscript.

Funding: This research was funded by CureKids NZ.

Acknowledgments: We thank the patients and their families described in this report. J.M. and S.P.R. are supported by Curekids, N.Z. The authors wish to acknowledge the contribution of NeSI high-performance computing facilities.

Conflicts of Interest: The authors have nothing to disclose. The authors declare no conflict of interest.

\section{References}

1. Vuillaume, M.L.; Valard, A.G.; Houcinat, N.; Bouron, J.; Boucher, C.; Deves, S.; Toutain, J.; Schaub, B.; Adenet, C.; Lacombe, D.; et al. First female prenatal case of osteopathia striata with cranial sclerosis in a fetus carrying a de-novo 1.9 Mbp interstitial deletion at Xq11.1q11.2. Clin. Dysmorphol. 2017, 26, 231-234. [CrossRef]

2. Enomoto, Y.; Tsurusaki, Y.; Harada, N.; Aida, N.; Kurosawa, K. Novel AMER1 frameshift mutation in a girl with osteopathia striata with cranial sclerosis. Congenit. Anom. (Kyoto) 2018, 58, 145-146. [CrossRef]

3. Comai, G.; Boutet, A.; Tanneberger, K.; Massa, F.; Rocha, A.S.; Charlet, A.; Panzolini, C.; Motamedi, F.J.; Brommage, R.; Hans, W.; et al. Genetic and Molecular Insights Into Genotype-Phenotype Relationships in Osteopathia Striata With Cranial Sclerosis (OSCS) Through the Analysis of Novel Mouse Wtx Mutant Alleles. J. Bone Min. Res. 2018, 33, 875-887. [CrossRef] [PubMed]

4. Holman, S.K.; Daniel, P.; Jenkins, Z.A.; Herron, R.L.; Morgan, T.; Savarirayan, R.; Chow, C.W.; Bohring, A.; Mosel, A.; Lacombe, D.; et al. The male phenotype in osteopathia striata congenita with cranial sclerosis. Am. J. Med. Genet. Part A 2011, 155, 2397-2408. [CrossRef] [PubMed]

5. Major, M.B.; Camp, N.D.; Berndt, J.D.; Yi, X.; Goldenberg, S.J.; Hubbert, C.; Biechele, T.L.; Gingras, A.N.; Zheng, N.; Maccoss, M.J.; et al. Wilms tumor suppressor WTX negatively regulates WNT/ $\beta$-catenin signaling. Science 2007, 316, 1043-1046. [CrossRef] [PubMed]

6. Sperling, M. Pediatric Endocrinology E-Book: Expert Consult-Online and Print; Elsevier Health Sciences: Philadelphia, PA, USA, 2014.

7. Maeda, K.; Kobayashi, Y.; Koide, M.; Uehara, S.; Okamoto, M.; Ishihara, A.; Kayama, T.; Saito, M.; Marumo, K. The Regulation of Bone Metabolism and Disorders by Wnt Signaling. Int. J. Mol. Sci. 2019, 20, 5525. [CrossRef] [PubMed]

8. Glorieux, F.H.; Pettifor, J.M.; Juppner, H. Pediatric Bone: Biology and Diseases; Elsevier Science: Cambridge, MA, USA, 2011.

9. Jenkins, Z.A.; van Kogelenberg, M.; Morgan, T.; Jeffs, A.; Fukuzawa, R.; Pearl, E.; Thaller, C.; Hing, A.V.; Porteous, M.E.; Garcia-Miñaur, S.; et al. Germline mutations in WTX cause a sclerosing skeletal dysplasia but do not predispose to tumorigenesis. Nat. Genet. 2009, 41, 95-100. [CrossRef] [PubMed]

10. McKenna, A.; Hanna, M.; Banks, E.; Sivachenko, A.; Cibulskis, K.; Kernytsky, A.; Garimella, K.; Altshuler, D.; Gabriel, S.; Daly, M.; et al. The Genome Analysis Toolkit: A MapReduce framework for analyzing next-generation DNA sequencing data. Genome Res. 2010, 20, 1297-1303. [CrossRef] [PubMed]

11. DePristo, M.A.; Banks, E.; Poplin, R.; Garimella, K.V.; Maguire, J.R.; Hartl, C.; Philippakis, A.A.; del Angel, G.; Rivas, M.A.; Hanna, M.; et al. A framework for variation discovery and genotyping using next-generation DNA sequencing data. Nat. Genet. 2011, 43, 91-498. [CrossRef] [PubMed]

12. Li, H.; Durbin, R. Fast and accurate short read alignment with Burrows-Wheeler transform. Bioinformatics 2009, 25, 1754-1760. [CrossRef] [PubMed]

13. Van der Auwera, G.A.; Carneiro, M.O.; Hartl, C.; Poplin, R.; del Angel, G.; Levy-Moonshine, A.; Jordan, T.; Shakir, K.; Roazen, D.; Thibault, J.; et al. From FastQ data to high-confidence variant calls: The genome analysis toolkit best practices pipeline. Curr. Protoc. Bioinform. 2013, 43, 11.10.1-11.10.33.

14. Cingolani, P.; Platts, A.; Wang, L.; Coon, M.; Nguyen, T.; Wang, L.; Land, S.J.; Lu, X.; Ruden, D.M. A program for annotating and predicting the effects of single nucleotide polymorphisms, SnpEff: SNPs in the genome of Drosophila melanogaster strain w1118; iso-2; iso-3. Fly 2012, 6, 80-92. [CrossRef] [PubMed]

15. Exome Aggregation Consortium. Analysis of protein-coding genetic variation in 60,706 humans. Nature 2015, 536, 285-291. 
16. Chen, X.; Schulz-Trieglaff, O.; Shaw, R.; Barnes, B.; Schlesinger, F.; Källberg, M.; Cox, A.J.; Kruglyak, S.; Saunders, C.T. Manta: Rapid detection of structural variants and indels for germline and cancer sequencing applications. Bioinformatics 2015, 32, 1220-1222. [CrossRef] [PubMed]

17. Richards, S.; Aziz, N.; Bale, S.; Bick, D.; Das, S.; Gastier-Foster, J.; Grody, W.W.; Hegde, M.; Lyon, E.; Spector, E.; et al. Standards and guidelines for the interpretation of sequence variants: A joint consensus recommendation of the American College of Medical Genetics and Genomics and the Association for Molecular Pathology. Genet. Med. 2015, 17, 405-424. [CrossRef] [PubMed]

Publisher's Note: MDPI stays neutral with regard to jurisdictional claims in published maps and institutional affiliations.

(C) 2020 by the authors. Licensee MDPI, Basel, Switzerland. This article is an open access article distributed under the terms and conditions of the Creative Commons Attribution (CC BY) license (http://creativecommons.org/licenses/by/4.0/). 